\title{
Feedback solutions of optimal control problems with DAE constraints *
}

\author{
Galina A. Kurina, Roswitha März
}

\begin{abstract}
An optimal feedback control has been obtained for linear-quadratic optimal control problems with constraints described by differential-algebraic equations. For that purpose, a new implicit Riccati equation (Riccati differential algebraic system) is provided, and its solvability is investigated. It is shown that one can do without those strong consistency conditions as used in several previous papers.

Also the solvability of the resulting closed loop system is considered and the relations between Riccati equations and Hamiltonian systems are elucidated.
\end{abstract}

Keywords: linear-quadratic optimal control problems, feedback control, differentialalgebraic equations, descriptor systems, Riccati equations, Hamiltonian systems

AMS subject classification: 49N10, 49J15, 49N35,34A09

\section{Introduction}

Feedback solutions via Riccati differential equations are a known and proved tool for solving linear quadratic optimal control problems given by the cost

$$
J(u, x):=\frac{1}{2}\langle x(T), V x(T)\rangle+\frac{1}{2} \int_{0}^{T}\left\langle\left[\begin{array}{l}
x(t) \\
u(t)
\end{array}\right],\left[\begin{array}{ll}
W(t) & S(t) \\
S(t)^{*} & R(t)
\end{array}\right]\left[\begin{array}{l}
x(t) \\
u(t)
\end{array}\right]\right\rangle d t
$$

and the side conditions

$$
\begin{gathered}
x^{\prime}(t)=C(t) x(t)+D(t) u(t), \quad t \in[0, T], \\
x(0)=z_{0} .
\end{gathered}
$$

Let all coefficients be continuous and certain standard conditions be fulfilled (cf. Section 2 ). The superscript $*$ denotes the transpose. In the following, the argument $t$ is dropped almost everywhere, and the given relations are ment pointwise for all $t \in[0, T]$.

The relevant Riccati matrix differential equation with the symmetric solution $Y$ is of the form

$$
Y^{\prime}=-Y C-C^{*} Y+(S+Y D) R^{-1}\left(S^{*}+D^{*} Y\right)-W
$$

*Supported by the DFG Research Center MATHEON "Mathematics for key technologies" 


$$
Y(T)=V \text {. }
$$

If the explicit ordinary differential equation (ODE) in (1.2) is replaced by a differential algebraic equation (DAE)

$$
E x^{\prime}=C x+D u,
$$

with $E$ being a singular constant square matrix, the situation becomes much more complex, and several different generalizations of the Riccati-ansatz are possible. For this, too, quite a lot of references are available (in particular for the case of constant coefficients), which, however, we can only partly mention here. We refer to [BeLa], [Ku4] and [KuMe] for further sources.

In $[\mathrm{BeLa}]$ it is first noted that the modification

$$
E^{*} Y^{\prime} E=-E^{*} Y C-C^{*} Y E+\left(S+E^{*} Y D\right) R^{-1}\left(S^{*}+D^{*} Y E\right)-W,
$$

which is considered to be obvious, leads to much too restrictive and unacceptable solvability conditions. Consequently, more specific Riccati approaches that skilfully make use of the inherent structures, find favour with [BeLa]. Starting from a singular value decomposition $U E V=\operatorname{diag}(\Sigma, 0)$ and certain rank conditions, lower dimensional Riccati equations of the form $\Sigma Y^{\prime} \Sigma=\ldots$ are introduced. From the point of view of DAE theory the rank conditions used in [BeLa] imply that the related Hamilton-Lagrange system is a regular DAE with tractability index one (cf. [BaKuMa]).

In [Ku1], [Ku2], [Ku3] (in in a more general Hilbert space setting, with $S=0$ ) a different ansatz was followed with Riccati equations of the form

$$
E^{*} Y^{\prime}=-Y^{*} C-C^{*} Y+\left(S+Y^{*} D\right) R^{-1}\left(S^{*}+D^{*} Y\right)-W .
$$

The solutions of the final value problem for (1.8) with the condition

$$
E^{*} Y(T)=V
$$

have the symmetry property $E^{*} Y=Y^{*} E$. Like (1.7), also (1.8) is primarily a matrixDAE, however, equation (1.8) has much better solvability properties than equation (1.7). In [Ku1], [Ku2], a decoupling into characteristic components is not used for the ansatz of the Riccati equation itself as it was done in [BeLa], but consistently for proving the solvability of the Riccati final value problem (1.8),(1.9) .

[KuMe] consider the Riccati DAE

$$
\left(E^{*} Y E\right)^{\prime}=-E^{*} Y C-C^{*} Y E+\left(S+E^{*} Y D\right) R^{-1}\left(S^{*}+D^{*} Y E\right)-W,
$$

which is a generalization of (1.7) for time-dependent coefficients $E$, however, without a positive result. The authors noticed that, unfortunately, this approach can only be used in very special cases since, for $E(t)$ singular, the solutions of (1.10) and the Euler-Lagrange equation are not related via $u=-R^{-1}\left(S+D^{*} Y E\right) x$ as in the case of nonsingular $E(t)$. If, in (1.6), there is no constant matrix $E$ in front of the derivative but a time-dependent one, it makes sense to change to a DAE with properly formulated leading term, i.e., instead of (1.6), to

$$
A(B x)^{\prime}=C x+D u,
$$

with well-matched $A$ and $B$. The corresponding initial condition is

$$
A(0) B(0) x(0)=z_{0}
$$


with $z_{0} \in \operatorname{im}(A(0) B(0))$ (cf. [BaMa]).

Under the assumption that $B$ is continuously differentiable, the Riccati equation

$$
\begin{gathered}
\left(B^{*} A^{*} Y\right)^{\prime}=-Y^{*}\left(C-A B^{\prime}\right)-\left(C^{*}-B^{* \prime} A^{*}\right) Y+\left(S+Y^{*} D\right) R^{-1}\left(S^{*}+D^{*} Y\right)-W, \\
B(T)^{*} A(T)^{*} Y(T)=V,
\end{gathered}
$$

with the symmetry property $B^{*} A^{*} Y=Y^{*} A B$ is proved to be relevant in [KlKu] (in a more general Hilbert space setting).

In [Do] the Riccati DAE

$$
B^{*}\left(A^{*} Y\right)^{\prime} B=-B^{*} Y^{*} C-C^{*} Y B+\left(S+B^{*} Y^{*} D\right) R^{-1}\left(S^{*}+D^{*} Y B\right)-W
$$

is investigated with only continuous $B$, which is, however, also a sort of generalization of (1.7) and adopts the bad solvability properties of (1.7). Under the condition that $\operatorname{ker} B^{*}=0$, the solutions of (1.14) with $B(T)^{*} A(T)^{*} Y(T) B(T)=V$ have the symmetry property $A^{*} Y=Y^{*} A$ and the ansatz $u=-R^{-1}\left(S^{*}+D^{*} Y B\right) x$ actually leads to an optimal feedback control. As already mentioned, this Riccati DAE involves practically unacceptable solution conditions.

In this paper we work with the Riccati DAE

$$
B^{*}\left(A^{*} Y B^{-}\right)^{\prime} B=-Y^{*} C-C^{*} Y+\left(S+Y^{*} D\right) R^{-1}\left(S^{*}+D^{*} Y\right)-W,
$$

and the final value condition

$$
A(T)^{*} Y(T) B(T)^{-}=B(T)^{-*} V B(T)^{-}
$$

doing without the assumption of smoothness of $B$. Here, the solutions fulfil the symmetry conditon $A^{*} Y B^{-}=B^{-*} Y^{*} A$ ( $B^{-}$is a special, generalized inverse).

Notice that (1.11) is no longer necessarily quadratic, but may contain $k$ equations while $x(t)$ has $m$ components.

In Section 2 it is shown that, for the linear quadratic optimal control problems (1.1),(1.11),(1.12), analogously to the classical case (1.1)-(1.5), optimal feedback controls can be established from the solutions of (1.15),(1.16). The main result in this respect is Theorem 2.5.

Section 3 investigates the solvability of the Riccati equation (1.15), generalizing the positive results from $[\mathrm{Ku} 2]$, [Ku3]. The obtained solvability statements are provided in Theorem 3.4 .

In Section 4 we show that the solvability assumptions from Theorem 3.4 simultaneously imply the solvability of the closed loop initial value problems.

Case studies in Section 5 show the better solvability properties of the new Riccati equation e.g. as compared to the properties from (1.7).

Finally, Section 6 elucidates the relation between solutions of the Riccati equation (1.15) and solutions of the corresponding implicit Hamiltonian system.

\section{Optimal feedback control}

We deal with the quadratic cost functional

$$
J(u, x):=\frac{1}{2}\langle x(T), V x(T)\rangle+\frac{1}{2} \int_{0}^{T}\{\langle x(t), W(t) x(t)\rangle+2\langle x(t), S(t) u(t)\rangle+\langle u(t), R(t) u(t)\rangle\} d t
$$


to be minimized on pairs $(u, x) \in C \times C_{B}^{1}$ satisfying the initial value problem (IVP)

$$
\begin{gathered}
A(t)(B(t) x(t))^{\prime}=C(t) x(t)+D(t) u(t), \quad t \in[0, T], \\
A(0) B(0) x(0)=z_{0} .
\end{gathered}
$$

The coefficients in (2.1),(2.2) are matrices $W(t) \in L\left(\mathbb{R}^{m}\right), R(t) \in L\left(\mathbb{R}^{l}\right), S(t) \in L\left(\mathbb{R}^{l}, \mathbb{R}^{m}\right)$, $A(t) \in L\left(\mathbb{R}^{n}, \mathbb{R}^{k}\right), B(t) \in L\left(\mathbb{R}^{m}, \mathbb{R}^{n}\right), C(t) \in L\left(\mathbb{R}^{m}, \mathbb{R}^{k}\right), D(t) \in L\left(\mathbb{R}^{l}, \mathbb{R}^{k}\right), t \in[0, T]$, which depend continuously on $t$, and $V \in L\left(\mathbb{R}^{m}\right)$.

The value $z_{0} \in i m(A(0) B(0))$ is given. The leading term of the DAE (2.2) is supposed to be properly stated in the sense that the decomposition

$$
\operatorname{ker} A(t) \oplus i m B(t)=\mathbb{R}^{n}, \quad t \in[0, T]
$$

holds true, and both subspaces forming this direct sum have constant dimensions and are spanned by continuously differentiable on $[0, T]$ base functions (cf. [BaMa]).

We use the symbols $C$ and $C^{1}$ for continuous resp. continuously differentiable function spaces (functions defined on $[0, T]$ with values in $\mathbb{R}^{l}, \mathbb{R}^{m}, \mathbb{R}^{k}$ or $\mathbb{R}^{n}$ as given by the context), and the denotations $C_{B}^{1}:=\left\{x \in C: B x \in C^{1}\right\}, C_{A^{*}}^{1}:=\left\{\psi \in C: A^{*} \psi \in C^{1}\right\}$.

The coefficients determining the cost (2.1) satisfy the following standard assumptions: $W(t), R(t)$ and $V$ are symmetric, $R(t)$ is positive definite, $\left[\begin{array}{ll}W(t) & S(t) \\ S(t)^{*} & R(t)\end{array}\right]$ is positive semidefinite, $t \in[0, T]$.

A pair $(u, x) \in C \times C_{B}^{1}$ satisfying the IVP (2.2),(2.3) is said to be admissible.

Let $K(t) \in L\left(\mathbb{R}^{n}\right)$ denote the projector that realizes decomposition $(2.4)$, $\operatorname{ker} K(t)=$ $\operatorname{ker} A(t), i m K(t)=i m B(t), t \in[0, T]$. Since these subspaces are continuously differentiable, so is the projector function $K:[0, T] \rightarrow L\left(\mathbb{R}^{n}\right)$.

Besides $K(t)$ we introduce $Q(t) \in L\left(\mathbb{R}^{m}\right), Q_{*}(t) \in L\left(\mathbb{R}^{k}\right)$ being the orthoprojectors onto $\operatorname{ker}(A(t) B(t))$ resp. onto $\operatorname{ker}\left(B(t)^{*} A(t)^{*}\right)=i m(A(t) B(t))^{\perp}$, further, $P(t):=I-$ $Q(t), P_{*}(t):=I-Q_{*}(t), t \in[0, T]$.

$Q, P, Q_{*}, P_{*}$ are continuous.

It is natural assuming $V=V P(T)$ (e.g. [KuMa]).

Having the projectors $K, P$ and $P_{*}$, we introduce the generalized inverses $B^{-}$of $B$ and $A^{*-}$ of $A^{*}$ by

$$
\begin{aligned}
& B^{-} B B^{-}=B^{-}, \quad B B^{-} B=B, \quad B B^{-}=K, \quad B^{-} B=P \\
& A^{*-} A^{*} A^{*-}=A^{*-}, \quad A^{*} A^{*-} A^{*}=A^{*}, \quad A^{*} A^{*-}=K^{*}, \quad A^{*-} A^{*}=P_{*} \text {. }
\end{aligned}
$$

Notice that $B^{-}$and $A^{*-}$ are uniquely determined by $(2.5)$, and continuous on $[0, T]$. It holds further simply

$$
B^{-} K=B^{-}, \quad A=A K, \quad A^{*}=K^{*} A^{*}, \quad B^{-*}=K^{*} B^{-*} .
$$

Next we consider the final value problem

$$
B^{*}\left(A^{*} Y B^{-}\right)^{\prime} B=-Y^{*} C-C^{*} Y+\left(S+Y^{*} D\right) R^{-1}\left(S^{*}+D^{*} Y\right)-W
$$




$$
A(T)^{*} Y(T) B(T)^{-}=B(T)^{-*} V B(T)^{-} .
$$

Equation (2.7) generalizes the (well-known for $A=I, B=I$ ) Riccati equation and may be understood as a Riccati-DAE.

Lemma 2.1 If $Y:[0, T] \rightarrow L\left(\mathbb{R}^{m}, \mathbb{R}^{k}\right)$ is continuous with a continuously differentiable part $A^{*} Y B^{-}$, and if it satisfies the IVP (2.7)(2.8), then the symmetry relation

$$
A^{*} Y B^{-}=B^{-*} Y^{*} A
$$

becomes true.

\section{Proof:}

Multiplying (2.7) by $B^{-*}$ from the left, and by $B^{-}$from the right leads to

$$
K^{*}\left(A^{*} Y B^{-}\right)^{\prime} K=B^{-*}\left\{-Y^{*} C-C^{*} Y+\left(S+Y^{*} D\right) R^{-1}\left(S^{*}+D^{*} Y\right)-W\right\} B^{-}=: \mathfrak{A},
$$

where $\mathfrak{A}=\mathfrak{A}^{*}$, and, further (cf. (2.6))

$$
\left(A^{*} Y B^{-}\right)^{\prime}=\mathfrak{A}+K^{*^{\prime}} A^{*} Y B^{-}+A^{*} Y B^{-} K^{\prime} .
$$

It becomes clear that $U:=A^{*} Y B^{-}$satisfies the ODE $U^{\prime}=\mathfrak{A}+K^{*^{\prime}} U+U K^{\prime}$ as well as the condition $U(T)=B(T)^{-*} V B(T)^{-}$. Obviously, $U^{*}$ is a further solution of the same IVP, i.e., $U=U^{*}$ must be true.

Remark 2.2 If $Y$ solves (2.7)(2.8) and if, additionally, the condition $A^{*} Y Q=0$ is given, then it follows that

$$
B^{*} A^{*} Y=Y^{*} A B
$$

must hold. Conversely, relation (2.10) implies $A^{*} Y Q=0$.

Remark 2.3 If one has, instead of a continuous coefficient $B$ considered here, a $B$ being continuously differentiable, one can consider

$$
\begin{gathered}
\left(B^{*} A^{*} Y\right)^{\prime}=B^{* \prime} A^{*} Y+Y^{*} A B^{\prime}-Y^{*} C-C^{*} Y+\left(S+Y^{*} D\right) R^{-1}\left(S^{*}+D^{*} Y\right)-W \\
B(T)^{*} A(T)^{*} Y(T)=P(T) V P(T)=V .
\end{gathered}
$$

instead of $(2.7)(2.8)$. All solutions of (2.11),(2.12) have the symmetry property (2.10). At the same time they are solutions of $(2.7),(2.8)$ and satisfy the additional condition $A^{*} Y Q=0$.

At first glance this shows that, considering the Riccati DAE (2.7), we are not confronted with those restrictive consistency conditions as we would be in case of (1.7),(1.10) and (1.14). On the contrary, we may expect positive results as in $[\mathrm{KlKu}]$.

Remark 2.4 Equation (2.11) was first considered in [KlKu]. Special cases resp. slight modifications of (2.11) were discussed in [Ku5] ( $A$ or $B$ is absent) and in [Ku1],[Ku2] ( $A$ is absent, $B$ is constant, $S=0$ ). 
Theorem 2.5 Let $Y$ be a solution of the IVP (2.7),(2.8), and let the condition $A^{*} Y Q=0$ be fulfilled.

Let $x_{*} \in C_{B}^{1}$ be a solution of the IVP

$$
A(B x)^{\prime}=C x-D R^{-1}\left(S^{*}+D^{*} Y\right) x, \quad A(0) B(0) x(0)=z_{0},
$$

and

$$
u_{*}:=-R^{-1}\left(S^{*}+D^{*} Y\right) x_{*} .
$$

Then, it holds for each admissible pair $(u, x) \in C \times C_{B}^{1}$ that

$$
J(u, x) \geq J\left(u_{*}, x_{*}\right)=\frac{1}{2}\left\langle z_{0}, A(0)^{*-} B(0)^{-*} Y(0)^{*} z_{0}\right\rangle,
$$

i.e., $\left(u_{*}, x_{*}\right)$ is an optimal pair and (2.14) describes the optimal feedback.

\section{Proof:}

It holds that $A^{*} Y=A^{*} Y P=A^{*} Y B^{-} B$, and $B^{-*} Y^{*} A B=A^{*} Y$. Given an admissible pair $(u, x)$, we derive

$$
\begin{aligned}
\frac{d}{d t}\left\langle B x, A^{*} Y x\right\rangle & =\left\langle(B x)^{\prime}, A^{*} Y x\right\rangle+\left\langle B x,\left(A^{*} Y B^{-} B x\right)^{\prime}\right\rangle \\
& =\left\langle(B x)^{\prime}, A^{*} Y x\right\rangle+\left\langle B x,\left(A^{*} Y B^{-}\right)^{\prime} B x\right\rangle+\left\langle B x, A^{*} Y B^{-}(B x)^{\prime}\right\rangle \\
& =\left\langle(B x)^{\prime}, A^{*} Y x\right\rangle+\left\langle B x,\left(A^{*} Y B^{-}\right)^{\prime} B x\right\rangle+\left\langle A^{*} Y x,(B x)^{\prime}\right\rangle \\
& =2\left\langle(B x)^{\prime}, A^{*} Y x\right\rangle+\left\langle x, B^{*}\left(A^{*} Y B^{-}\right)^{\prime} B x\right\rangle \\
& =2\left\langle A(B x)^{\prime}, Y x\right\rangle+\left\langle x, B^{*}\left(A^{*} Y B^{-}\right)^{\prime} B x\right\rangle .
\end{aligned}
$$

Taking into account the equations (2.2) and (2.7) we obtain the expression

$$
\begin{aligned}
\frac{d}{d t}\left\langle B x, A^{*} Y x\right\rangle= & -\{\langle W x, x\rangle+2\langle S u, x\rangle+\langle R u, u\rangle\} \\
& +\left\langle R\left(u+R^{-1}\left(S^{*} x+D^{*} Y x\right)\right), u+R^{-1}\left(S^{*} x+D^{*} Y x\right)\right\rangle .
\end{aligned}
$$

By this we find

$$
\begin{gathered}
J(u, x)=\frac{1}{2}\langle x(T), V x(T)\rangle-\frac{1}{2} \int_{0}^{T} \frac{d}{d t}\left\langle B(t) x(t), A(t)^{*} Y(t) x(t)\right\rangle d t+\mathfrak{B}(u, x), \\
\mathfrak{B}(u, x)=\frac{1}{2} \int_{0}^{T}\left\langle R(t)\left(u(t)+R(t)^{-1}\left(S(t)^{*}+D(t)^{*} Y(t)\right) x(t)\right), u(t)+\right. \\
\left.+R(t)^{-1}\left(S(t)^{*}+D(t)^{*} Y(t)\right) x(t)\right\rangle d t .
\end{gathered}
$$

From the positive definiteness of $R(t)$ it follows that $\mathfrak{B}(u, x) \geq 0$.

Notice that $\mathfrak{B}\left(u_{*}, x_{*}\right)=0$.

Compute further

$$
\begin{aligned}
J(u, x)= & \frac{1}{2}\langle x(T), V x(T)\rangle-\frac{1}{2}\left\langle B(T) x(T), A(T)^{*} Y(T) x(T)\right\rangle \\
& +\frac{1}{2}\left\langle B(0) x(0), A(0)^{*} Y(0) x(0)\right\rangle+\mathfrak{B}(u, x) .
\end{aligned}
$$


Using the conditions (2.3) and (2.8) as well as the relations $V=V P(T), A^{*} Y=A^{*} Y B^{-} B$ and (2.10) we arrive at

$$
J(u, x)=\frac{1}{2}\left\langle z_{0}, A(0)^{*-} B(0)^{-*} Y(0)^{*} z_{0}\right\rangle+\mathfrak{B}(u, x) .
$$

Since the first term is independent of the admissible pair $(u, x)$, we conclude

$$
J(u, x) \geq \frac{1}{2}\left\langle z_{0}, A(0)^{*-} B(0)^{-*} Y(0)^{*} z_{0}\right\rangle=J\left(u_{*}, x_{*}\right) .
$$

\section{Solvability of the Riccati-DAE system}

In this section we consider solutions of the system

$$
\begin{gathered}
B^{*}\left(A^{*} Y B^{-}\right)^{\prime} B=-Y^{*} C-C^{*} Y+\left(S+Y^{*} D\right) R^{-1}\left(S^{*}+D^{*} Y\right)-W \\
P_{*} Y Q=0
\end{gathered}
$$

which satisfy the final condition

$$
A(T)^{*} Y(T) B(T)^{-}=\widetilde{V}:=B(T)^{-*} V B(T)^{-}
$$

Each solution $Y$ that must be continuous with a continuously differentiable part $A^{*} Y B^{-}$ can be decomposed as

$$
\begin{aligned}
Y & =P_{*} Y P+Q_{*} Y P+Q_{*} Y Q \\
& =A^{*-} A^{*} Y B^{-} B+Q_{*} Y P+Q_{*} Y Q
\end{aligned}
$$

We are going to show that the components

$$
U:=A^{*} Y B^{-} \in C^{1}\left([0, T], L\left(\mathbb{R}^{n}\right)\right), \quad \mathcal{V}:=Q_{*} Y P, \quad Z:=Q_{*} Y Q=Y Q \in C\left([0, T], L\left(\mathbb{R}^{m}, \mathbb{R}^{k}\right)\right)
$$

satisfy a standard Riccati differential equation, a linear equation, and an algebraic Riccati equation, respectively.

Multiplying (3.1) by $Q$ from the left and right, then by $Q$ from the left and $P$ from the right, and also by $B^{-*}$ from the left and $B^{-}$from the right, we obtain the system

$$
\begin{aligned}
& 0=-(Y Q)^{*} C Q-Q C^{*} Y Q+\left(Q S+(Y Q)^{*} D\right) R^{-1}\left(S^{*} Q+D^{*} Y Q\right)-Q W Q, \\
& 0=-(Y Q)^{*} C P-Q C^{*} Y P+\left(Q S+(Y Q)^{*} D\right) R^{-1}\left(S^{*} P+D^{*} Y P\right)-Q W P, \\
& K^{*}\left(A^{*} Y B^{-}\right)^{\prime} K=-\left(Y B^{-}\right)^{*} C B^{-}-B^{-*} C^{*} Y B^{-} \\
& +\left(B^{-*} S+\left(Y B^{-}\right)^{*} D\right) R^{-1}\left(S^{*} B^{-}+D^{*} Y B^{-}\right)-B^{-*} W B^{-} \text {. }
\end{aligned}
$$


Since multiplication of (3.1) by $P$ from the left and $Q$ from the right yields once more equation (3.6), we know (3.1) to be equivalent to (3.5)- (3.7). Obviously, the component $Z=Q_{*} Y Q=Y Q$ satisfies (cf. (3.5)) the algebraic Riccati equation

$$
0=-Z^{*} Q_{*} C Q-Q C^{*} Q_{*} Z+\left(Q S+Z^{*} Q_{*} D\right) R^{-1}\left(S^{*} Q+D^{*} Q_{*} Z\right)-Q W Q,
$$

and the trivial conditons $P_{*} Z=0, Z P=0$.

Next, from (3.6) we obtain a linear relation for the components $Z, U$ and $\mathcal{V}$, namely

$$
M Q_{*} \mathcal{V}+M P_{*} A^{*-} U B=-Z^{*} Q_{*} C P+\left(Q S+Z^{*} Q_{*} D\right) R^{-1} S^{*} P-Q W P,
$$

where

$$
M:=Q C^{*}-\left(Q S+Z^{*} Q_{*} D\right) R^{-1} D^{*}, \quad M=Q M .
$$

Notice that, if the conditions

$$
i m M Q_{*}=i m Q, \quad \operatorname{ker} M \cap i m Q_{*}=0
$$

are fulfilled, we have also $\operatorname{ker} M Q_{*}=\operatorname{ker} Q_{*}$, further

$$
\left(M Q_{*}\right)^{+} M Q_{*}=Q_{*}, \quad M Q_{*}\left(M Q_{*}\right)^{+}=Q,
$$

and the resulting linear equation

$$
M Q_{*} \mathcal{V}=-Z^{*} Q_{*} C P+\left(Q S+Z^{*} Q_{*} D\right) R^{-1} S^{*} P-Q W P-M P_{*} A^{*-} U B,
$$

determines $\mathcal{V}$ uniquely depending on $Z$ and $U$. Let us write then

$$
\mathcal{V}=C_{1}+C_{2} A^{*-} U B
$$

with

$$
\begin{aligned}
& C_{1}:=\left(M Q_{*}\right)^{+}\left\{-Z^{*} Q_{*} C P+\left(Q S+Z^{*} Q_{*} D\right) R^{-1} S^{*} P-Q W P\right\}, \\
& C_{2}:=-\left(M Q_{*}\right)^{+} M P_{*}
\end{aligned}
$$

Notice that $\left(M Q_{*}\right)^{+}$is continuous. It holds that $C_{1}=Q_{*} C_{1}=C_{1} P, C_{2}=Q_{*} C_{2}=C_{2} P_{*}$. Finally we turn to (3.7). Since $K$ is continuously differentiable and $U K=U, K^{*} U=U$ hold true, we may write

$$
K^{*}\left(A^{*} Y B^{-}\right)^{\prime} K=K^{*} U^{\prime} K=U^{\prime}-K^{* \prime} U-U K^{\prime}
$$

Recall that $U$ is symmetric due to Lemma 2.1. Using (3.13) we derive

$$
\begin{aligned}
Y P & =Q_{*} Y P+P_{*} Y P=\mathcal{V}+A^{*-} U B \\
& =C_{1}+C_{2} A^{*-} U B+A^{*-} U B,
\end{aligned}
$$

that is,

$$
Y P=C_{1}+C_{3} A^{*-} U B, \quad C_{3}:=C_{2}+P_{*} .
$$

Thus we obtain, from (3.7), the following differential equation for $U$

$$
\begin{aligned}
U^{\prime}= & K^{* \prime} U+U^{*} K^{\prime}-B^{-*} W B^{-}-B^{-*}\left(C_{1}+C_{3} A^{*-} U B\right)^{*} C B^{-} \\
& -B^{-*} C^{*}\left(C_{1}+C_{3} A^{*-} U B\right) B^{-} \\
& +B^{-*}\left(S+\left(C_{1}+C_{3} A^{*-} U B\right)^{*} D\right) R^{-1}\left(S^{*}+D^{*}\left(C_{1}+C_{3} A^{*-} U B\right)\right) B^{-},
\end{aligned}
$$


that is, considering that $U$ is symmetric,

$$
U^{\prime}=-\widetilde{W}-\widetilde{C}^{*} U-U \widetilde{C}+U \widetilde{D} R^{-1} \widetilde{D}^{*} U
$$

where

$$
\begin{aligned}
\widetilde{C}^{*}:= & -K^{* \prime}+B^{-*} C^{*} C_{3} A^{*-}-B^{-*}\left(S+C_{1}^{*} D\right) R^{-1} D^{*} C_{3} A^{*-}, \\
\widetilde{D}^{*}:= & D^{*} C_{3} A^{*-}, \\
\widetilde{W}:= & B^{-*}\left\{P W P+P C_{1}^{*} C P+P C^{*} C_{1} P\right. \\
& \left.\quad-P\left(S+C_{1}^{*} D\right) R^{-1}\left(S^{*}+D^{*} C_{1}\right) P\right\} B^{-}=\widetilde{W^{*}} .
\end{aligned}
$$

Lemma 3.1 Let condition (3.10) be given, and additionally,

$$
i m Z=i m Q_{*}, \quad \operatorname{ker} Z=\operatorname{ker} Q .
$$

Then, equation (3.15) represents a standard Riccati differential equation with a symmetric, positive semidefinite coefficient $\widetilde{W}$.

\section{Proof:}

Condition (3.16) leads to $Z Z^{+}=Q_{*}, Z^{+} Z=Q$, and $Z^{+}$is continuous.

By construction of $C_{2}, C_{1}$ it holds that

$$
\begin{aligned}
M Q_{*} C_{2} & =-Q M P_{*}=-M P_{*}, \\
M P_{*} A^{*-} U B & =-M Q_{*} C_{2} A^{*-} U B=-M Q_{*}\left(\mathcal{V}-C_{1}\right)=M Q_{*} C_{1}-M Q_{*} \mathcal{V}
\end{aligned}
$$

Taking this into account, we obtain from (3.12) the relation

$$
0=-Q W P-Z^{*} Q_{*} C P+\left(Q S+Z^{*} Q_{*} D\right) R^{-1} S^{*} P-M Q_{*} C_{1} .
$$

Next we turn to

$$
M Q_{*}=Q C^{*} Q_{*}-\left(Q S+Z^{*} Q_{*} D\right) R^{-1} D^{*} Q_{*} .
$$

From (3.8) we derive the expression

$$
\begin{aligned}
\left(Q S+Z^{*} Q_{*} D\right) R^{-1} D^{*} Q_{*}= & Q W Q Z^{+}+Z^{*} Q_{*} C Q Z^{+}+Q C^{*} Q_{*} \\
& -\left(Q S+Z^{*} Q_{*} D\right) R^{-1} S^{*} Q Z^{+}
\end{aligned}
$$

and put it into the formula for $M Q_{*}$, that is

$$
M Q_{*}=-Q W Q Z^{+}-Z^{*} Q_{*} C Q Z^{+}+\left(Q S+Z^{*} Q_{*} D\right) R^{-1} S^{*} Q Z^{+} .
$$

By this, (3.17) becomes

$$
\begin{aligned}
0=-Q W P & -Z^{*} Q_{*} C P+\left(Q S+Z^{*} Q_{*} D\right) R^{-1} S^{*} P \\
& +Q W Q Z^{+} C_{1}+Z^{*} Q_{*} C Q Z^{+} C_{1}-\left(Q S+Z^{*} Q_{*} D\right) R^{-1} S^{*} Q Z^{+} C_{1},
\end{aligned}
$$

hence, by multiplication from the left by $C_{1}^{*} Z^{+*}$,

$$
\begin{aligned}
0=- & C_{1}^{*} Z^{+*} Q W P-C_{1}^{*} Q_{*} C P+C_{1}^{*} Z^{+*}\left(Q S+Z^{*} Q_{*} D\right) R^{-1} S^{*}\left(P-Q Z^{+} C_{1}\right) \\
& +C_{1}^{*} Z^{+*} Q W Q Z^{+} C_{1}+C_{1}^{*} Q_{*} C Q Z^{+} C_{1} .
\end{aligned}
$$


This yields the expressions

$$
\begin{aligned}
C_{1}^{*} C P= & C_{1}^{*} Q_{*} C P=-C_{1}^{*} Z^{+*} Q W P+C_{1}^{*} Q_{*} C Q Z^{+} C_{1} \\
& +C_{1}^{*} Z^{+*} Q W Q Z^{+} C_{1}+C_{1}^{*} Z^{+*}\left(Q S+Z^{*} Q_{*} D\right) R^{-1} S^{*}\left(P-Q Z^{+} C_{1}\right),
\end{aligned}
$$

and, using properties of $C_{1}$,

$$
\begin{aligned}
B^{*} \widetilde{W} B= & P W P+P C_{1}^{*} C P+P C^{*} C_{1} P-P\left(S+C_{1}^{*} D\right) R^{-1}\left(S^{*}+D^{*} C_{1}\right) P \\
= & P W P+C_{1}^{*} Q_{*} C P+P C^{*} Q_{*} C_{1}-\left(P S+C_{1}^{*} D\right) R^{-1}\left(S^{*} P+D^{*} C_{1}\right) \\
= & P W P-P S R^{-1} S^{*} P-P S R^{-1} D^{*} C_{1}-C_{1}^{*} D R^{-1} S^{*} P-C_{1}^{*} D R^{-1} D^{*} C_{1} \\
& -C_{1}^{*} Z^{+*} Q W P+C_{1}^{*} Q_{*} C Q Z^{+} C_{1}+C_{1}^{*} Z^{+*} Q W Q Z^{+} C_{1} \\
& +C_{1}^{*} Z^{+*} Q S R^{-1} S^{*} P-C_{1}^{*} Z^{+*} Q S R^{-1} S^{*} Q Z^{+} C_{1} \\
& +C_{1}^{*} Q_{*} D R^{-1} S^{*} P-C_{1}^{*} Q_{*} D R^{-1} S^{*} Q Z^{+} C_{1} \\
& -P W Q Z^{+} C_{1}+C_{1}^{*} Z^{+*} Q C^{*} Q_{*} C_{1}+C_{1}^{*} Z^{+*} Q W Q Z^{+} C_{1} \\
& +P S R^{-1} S^{*} Q Z^{+} C_{1}-C_{1}^{*} Z^{+*} Q S R^{-1} S^{*} Q Z^{+} C_{1} \\
& +P S R^{-1} D^{*} Q_{*} C_{1}-C_{1}^{*} Z^{+*} Q S R^{-1} D^{*} Q_{*} C_{1} \\
= & \left(P-C_{1}^{*} Z^{+*} Q\right)\left(W-S R^{-1} S^{*}\right)\left(P-Q Z^{+} C_{1}\right)+\mathfrak{B}, \\
\mathfrak{B}= & -C_{1}^{*} D R^{-1} D^{*} C_{1}+C_{1}^{*} Q_{*} C Q Z^{+} C_{1}+C_{1}^{*} Z^{+*} Q W Q Z^{+} C_{1} \\
& -C_{1}^{*} Q_{*} D R^{-1} S^{*} Q Z^{+} C_{1}+C_{1}^{*} Z^{+*} Q C^{*} Q_{*} C_{1} \\
& -C_{1}^{*} Z^{+*} Q S R^{-1} S^{*} Q Z^{+} C_{1}-C_{1}^{*} Z^{+*} Q S R^{-1} D^{*} Q_{*} C_{1} .
\end{aligned}
$$

Because of (cf. (3.8)

$$
\begin{array}{r}
C_{1}^{*} Z^{+*} Q W Q Z^{+} C_{1}+C_{1}^{*} Q_{*} C Q Z^{+} C_{1}+C_{1}^{*} Z^{+*} Q C^{*} Q_{*} C_{1} \\
\quad=C_{1}^{*} Z^{+*}\left(Q S+Z^{*} Q_{*} D\right) R^{-1}\left(S^{*} Q+D^{*} Q_{*} Z\right) Z^{+} C_{1}
\end{array}
$$

we find

$$
\begin{aligned}
\mathfrak{B}:= & -C_{1}^{*} D R^{-1} D^{*} C_{1}-C_{1}^{*} Q_{*} D R^{-1} S^{*} Q Z^{+} C_{1}-C_{1}^{*} Z^{+*} Q S R^{-1} S^{*} Q Z^{+} C_{1} \\
& -C_{1}^{*} Z^{+*} Q S R^{-1} D^{*} Q_{*} C_{1} \\
& +C_{1}^{*} Z^{+*} Q S R^{-1} S^{*} Q Z^{+} C_{1}+C_{1}^{*} Z^{+*} Q S R^{-1} D^{*} Q_{*} C_{1} \\
& +C_{1}^{*} Q_{*} D R^{-1} S^{*} Q Z^{+} C_{1}+C_{1}^{*} Q_{*} D R^{-1} D^{*} Q_{*} C_{1}=0 .
\end{aligned}
$$

It results that

$$
\widetilde{W}=B^{-*}\left(P-C_{1}^{*} Z^{+*} Q\right)\left(W-S R^{-1} S^{*}\right)\left(P-Q Z^{+} C_{1}\right) B^{-} .
$$

For $t \in[0, T]$ and all $x \in \mathbb{R}^{m}$ it holds that

$$
\begin{aligned}
& \left\langle\left(W(t)-S(t) R(t)^{-1} S(t)^{*}\right) x, x\right\rangle= \\
& \quad\left\langle\left[\begin{array}{ll}
W(t) & S(t) \\
S(t)^{*} & R(t)
\end{array}\right]\left[\begin{array}{l}
x \\
-R(t)^{-1} S(t)^{*} x
\end{array}\right],\left[\begin{array}{l}
x \\
-R(t)^{-1} S(t)^{*} x
\end{array}\right]\right\rangle \geq 0,
\end{aligned}
$$

i.e., $W(t)-S(t) R(t)^{-1} S(t)^{*}$ is positive semidefinite, and so is $\widetilde{W}(t)$.

The following assertion reflects what we derived so far. 
Theorem 3.2 If $Y$ is a solution of the Riccati type final value problem (3.1), (3.2),(3.3), and if the conditions (3.10) and (3.16) are fulfilled, then the component $Z=Q_{*} Y Q$ is a solution of the algebraic Riccati equation (3.8), $U=A^{*} Y B^{-}$is a solution of the standard Riccati differential equation (3.15), and $\mathcal{V}=Q_{*} Y P$ satisfies (3.12).

Conversely, considering now the following decoupled system for the unknown functions $Z, U, \mathcal{V}$ to be given (cf. (3.8),(3.2), (3.15),(2.8),(3.12)) as

$$
\begin{gathered}
0=-Z^{*} Q_{*} C Q-Q C^{*} Q_{*} Z+\left(Q S+Z^{*} Q_{*} D\right) R^{-1}\left(S^{*} Q+D^{*} Q_{*} Z\right)-Q W Q, \\
P_{*} Z=0, \\
Z P=0 \\
U^{\prime}=-\widetilde{W}-\widetilde{C}^{*} U-U^{*} \widetilde{C}+U^{*} \widetilde{D} R^{-1} \widetilde{D}^{*} U \\
U(T)=\widetilde{V}:=B(T)^{-*} V B(T)^{-}, \\
M Q_{*} \mathcal{V}=-M P_{*} A^{*-} U B-Q W P-Z^{*} Q_{*} C P+\left(Q S+Z^{*} Q_{*} D\right) R^{-1} S^{*} P,
\end{gathered}
$$

we may try and compose a solution $Y$ of the original Riccati system (3.1)-(3.3) from the solutions $Z, U, \mathcal{V}$. Let us recall that the coefficients $\widetilde{C}, \widetilde{D}$ and $M$ as defined above depend on $Z$.

If $Z$ is a solution of the algebraic equation (3.19), then $Z+P_{*} \widetilde{Z}, \widetilde{Z}$ an arbitrary $k \times m$ matrix function, is also a solution of (3.19). By means of (3.20), the arbitrary solution part belonging to $i m P_{*}$ is fixed to be zero.

By multiplication of (3.8) from both sides by $Q$ we realize that, if $Z$ solves (3.8), then also $Z Q$ does so. By means of condition (3.21) we pick up solutions with $Z=Z Q$. From (3.20),(3.21) we have $Z=Q_{*} Z Q$.

Obviously, equation (3.19) itself is symmetric, but $Z$ is not so necessarily. Notice that $Z$ has $k$ rows and $m$ columns. If $m=k$, and $Q_{*}=Q$ (i.e. $\left.\operatorname{ker} A B=\operatorname{ker}(A B)^{*}\right)$, then $Z$ can be expected to be symmetric.

What we need is a continuous solution $Z$ that satisfies the conditions

$$
\begin{gathered}
i m Z=i m Q_{*}, \quad k e r Z=k \operatorname{er} Q, \\
i m M Q_{*}=i m Q, \quad k e r M Q_{*}=\operatorname{ker} Q_{*},
\end{gathered}
$$

with $M=Q C^{*}-\left(Q S+Z^{*} Q_{*} D\right) R^{-1} D^{*}$.

These requirements ensure the coefficients $\widetilde{W}, \widetilde{C}$ and $\widetilde{D}$ in (3.22) to be well-defined and continuous. Additionally, $\widetilde{W}$ is symmetric and positive semidefinite. It comes out that (3.22) is a standard Riccati differential equation, and the solution $U$ of the final value problem $(3.22),(3.23)$ is symmetric, $U=U^{*}$.

Lemma 3.3 Given a continuous solution $Z$ of (3.19)-(3.21) such that the conditions (3.25),(3.26) are fulfilled. Then, for the unique solution $U$ of the resulting standard Riccati differential equation (3.22), which has the final value given by (3.23), the relations

$$
U=U^{*}, \quad U=U K, \quad U=K^{*} U K
$$

hold true. 


\section{Proof:}

Let $U$ be a solution of (3.22),(3.23). It remains to verify that $U=K^{*} U K$.

Inspecting the coeffcients we find that $\widetilde{W}(I-K)=0, \widetilde{D}=K \widetilde{D}, \widetilde{C}=-K^{\prime}+K \widetilde{C} K$ must hold. Multiplying (3.22) by $(I-K)$ from the right hand side, we derive

$$
U^{\prime}(I-K)=-\widetilde{C}^{*} U(I-K)-U^{*}\left(-K^{\prime}+K \widetilde{C} K\right)(I-K)+U^{*} K \widetilde{D} R^{-1} \widetilde{D}^{*} U(I-K),
$$

hence, denoting $U(I-K)=: \widetilde{U}, U K=: U_{K}$, and taking into account that $U=U^{*}$,

$$
\widetilde{U}^{\prime}-U(I-K)^{\prime}=-\widetilde{C}^{*} \widetilde{U}+U K^{\prime}(I-K)+U_{K} \widetilde{D} R^{-1} \widetilde{D}^{*} \widetilde{U}
$$

i.e.,

$$
\begin{aligned}
\widetilde{U}^{\prime} & =U(I-K)^{\prime}-U K(I-K)^{\prime}+\left(U_{K} \widetilde{D} R^{-1} \widetilde{D}^{*}-\widetilde{C}^{*}\right) \widetilde{U} \\
& =\widetilde{U}(I-K)^{\prime}+F \widetilde{U} \\
F & :=U_{K} \widetilde{D} R^{-1} \widetilde{D}^{*}-\widetilde{C}^{*} .
\end{aligned}
$$

It becomes clear that the function $\widetilde{U}=U(I-K)$ is the solution of the homogeneous linear final value problem $\widetilde{U}^{\prime}=-\widetilde{U} K^{\prime}+F \widetilde{U}, \widetilde{U}(T)=0$, but then $\widetilde{U}$ vanishes identically. $\widetilde{U}=0$ means $U=U K$, further $U=U^{*}=K^{*} U=K^{*} U K$.

Having the matrix functions $U$ and $Z$ we compose

$$
\mathcal{V}:=\left(M Q_{*}\right)^{+}\left\{-M P_{*} A^{*-} U B+\left(Q S+Z^{*} Q_{*} D\right) R^{-1} S^{*} P-Q W P-Z^{*} Q_{*} C P\right\},
$$

to satisfy (3.24) and, finally

$$
Y:=A^{*-} U B+Z+\mathcal{V}
$$

Under the assumptions of Lemma 3.3, both $\mathcal{V}$ and $Y$ are continuous. It holds that

$$
Q_{*} Y P=Q_{*} \mathcal{V} P=\mathcal{V}, \quad Q_{*} Y Q=Q_{*} Z Q=Z, \quad A^{*} Y B^{-}=K^{*} U K=U .
$$

The component $A^{*} Y B^{-}$of $Y$ is continuously differentiable and symmetric. Straightforward calculations in the opposite direction to that we realized to provide system (3.19)(3.24) will show $Y$ to be a solution of our system (3.1)-(3.3). By this, the following assertion providing the solution $Y$ for Theorem 2.5 is proved.

Theorem 3.4 Let the algebraic Riccati system (3.19)-(3.21) have a continuous solution $Z$ that satisfies the conditions (3.25) and (3.26).

Then, the original Riccati-DAE system (3.1)-(3.3) has a continuous solution $Y$ whose component $A^{*} Y B^{-}$is continuously differentiable and symmetric. Additionally, it holds that $A^{*} Y Q=0$.

Remark 3.5 For special solvability assertions concerning algebraic Riccati equations as well as standard Riccati differential equations we refer to [A-K et all]. 


\section{Solvability of the closed loop problem}

To benefit from Theorem 2.5, besides the solution $Y$ of the Riccati-DAE one needs a solution of the resulting IVP (cf. (2.13)).

$$
\begin{gathered}
A(B x)^{\prime}=C x-D R^{-1}\left(S^{*}+D^{*} Y\right) x \\
A(0) B(0) x(0)=z_{0}
\end{gathered}
$$

where $z_{0} \in i m(A(0) B(0))$ is given.

Theorem 4.1 Let the conditions of Theorem 3.4 be given, $m=k$, and $Y$ be the solution of the Riccati-DAE system (3.1)-(3.3). Then the DAE (4.1) is regular with tractability index one, and there is exactly one solution $x_{*} \in C_{B}^{1}$ of the IVP (4.1),(4.2).

\section{Proof:}

As it is e.g. shown in [BaMa], the IVP-solvability is a consequence of the index-one property. Notice that a linear DAE with properly stated leading term is regular with index one if its adjoint equation is so, and vice versa (e.g. [BaMa]). The adjoint equation to $(4.1)$ reads

$$
-B^{*}\left(A^{*} \lambda\right)^{\prime}=C^{*} \lambda-\left(Y^{*} D+S\right) R^{-1} D^{*} \lambda .
$$

The DAE (4.3) is regular with index one if $\operatorname{ker} B^{*} A^{*}=i m Q_{*}$ and $\operatorname{ker} Q\left\{C^{*}-\left(Y^{*} D+\right.\right.$ $\left.S) R^{-1} D^{*}\right\}=: S_{*}$ intersect trivially (e.g. [BaMa]). Because of $Q Y^{*}=(Y Q)^{*}=(Z Q)^{*}=$ $Q Z^{*}$ we have, at the same time, $S_{*}=\operatorname{ker}\left\{Q C^{*}-\left(Q Z^{*} D+Q S\right) R^{-1} D^{*}\right\}=\operatorname{ker} M$. This means, the DAE (4.3) is regular with index one if $\operatorname{ker} M$ and $i m Q_{*}$ intersect trivially, but this in turn is a consequence of condition (3.26).

Theorem 4.2 Let the conditions of Theorem 3.4 be given, $m>k$ and $Y$ be the solution of the Riccati-DAE system (3.1)-(3.3). Then there are solutions $x_{*} \in C_{B}^{1}$ of the IVP (4.1),(4.2)

\section{Proof:}

Compute $G_{1}:=A B-\left\{C-D R^{-1}\left(S^{*}+D^{*} Y\right)\right\} Q$ and ask whether this matrix function has full row rank $k$. Obviously, this is in fact the case if $Q_{*}\left\{C Q-D R^{-1}\left(S^{*} Q+D^{*} Z Q\right)\right\}=$ $Q_{*} M^{*}=\left(M Q_{*}\right)^{*}$ has the same range as $Q_{*}$, i.e., if $i m\left(M Q_{*}\right)^{*}=i m Q_{*}$. However, this is ensured by (3.26).

Denote $F:=C-D R^{-1}\left(S^{*}+D^{*} Y\right)$.

In [CaMa], Proof of Proposition 3.2, a coordinate transform $x=H \bar{x}$ is applied to the DAE $A(B x)^{\prime}=F x$ with full row rank $G_{1}=A B-F Q$ such that the transformed variable has the structure $\left.\bar{x}=\left(\begin{array}{c}z \\ v\end{array}\right)\right\} m-k$ and the transformed IVP is of the form

$$
A(\widetilde{B} z)^{\prime}=\widetilde{F}_{1} z+\widetilde{F}_{2} v, \quad A(0) \widetilde{B}(0) z(0)=z_{0}
$$

while (4.4), with any given $v$, represents a regular index one DAE for $z$.

Remark 4.3 By fixing $v$ in (4.4), the resulting IVP for $z$ is uniquely solvable. How to choose the mentioned transformation $H$ in practice is discussed in [CaMa]. 


\section{A case study}

Here we deal with the very special case if $k=m=2, \quad n=1, \quad l=1, \quad T=1$,

$$
J(u, x)=\frac{1}{2} \int_{0}^{1}\left(\alpha x_{1}(t)^{2}+\beta x_{2}(t)^{2}+u(t)^{2}\right) d t,
$$

where $\alpha \geq 0, \beta \geq 0$, i.e., $W=\left(\begin{array}{ll}\alpha & 0 \\ 0 & \beta\end{array}\right), \quad R=1, V=0, S=0$, and the DAE describing the side condition is

$$
\begin{aligned}
x_{1}^{\prime}(t) & =c_{12}(t) x_{2}(t) \\
0 & =c_{21}(t) x_{1}(t)+c_{22}(t) x_{2}(t)+u(t)
\end{aligned}
$$

i.e., $A=\left[\begin{array}{l}1 \\ 0\end{array}\right], B=\left[\begin{array}{ll}1 & 0\end{array}\right], B^{-}=\left[\begin{array}{l}1 \\ 0\end{array}\right], K=1, D=\left[\begin{array}{l}0 \\ 1\end{array}\right], C=\left[\begin{array}{cc}0 & c_{12} \\ c_{21} & c_{22}\end{array}\right]$. The initial condition for (5.2) reads

$$
x_{1}(0)=x_{10} .
$$

We have taken this problem from [KuMe] and will discuss the same three cases in the following. We should like to point out that this little scholastic task (resp. special cases and infinite-horizon modifications) was already used earlier for illustative purposes in the literarure (e.g. [BeLa]).

Consider the Riccati-DAE system (3.1)-(3.3) for the $2 \times 2$ matrix function $Y=\left[\begin{array}{ll}Y_{11} & Y_{12} \\ Y_{21} & Y_{22}\end{array}\right]$. We describe (3.1) by means of the three equations (cf. (3.5), (3.6),(3.7)), taking into account that we have here $Q=Q_{*}=\left[\begin{array}{ll}0 & 0 \\ 0 & 1\end{array}\right], P=P_{*}=\left[\begin{array}{ll}1 & 0 \\ 0 & 0\end{array}\right]$, and dropping the equations " $0=0 "$,

$$
\begin{gathered}
0=-\beta-\left(Y_{12} c_{12}+Y_{22} c_{22}\right)-\left(c_{12} Y_{12}+c_{22} Y_{22}\right)+Y_{22}^{2} \\
0=Y_{22} Y_{21}-c_{21} Y_{22}-\left(c_{12} Y_{11}+c_{22} Y_{21}\right) \\
Y_{11}^{\prime}=-\alpha-c_{21} Y_{21}-c_{21} Y_{21}+Y_{21}^{2}
\end{gathered}
$$

The final value condition (3.3) is

$$
Y_{11}(1)=0
$$

and condition (3.2) means here

$$
Y_{12}=0
$$

By the use of (5.8), (5.4) simplifies to

$$
0=-\beta+\left(Y_{22}-c_{22}\right)^{2}-c_{22}^{2}
$$

This algebraic equation has the solutions

$$
Y_{22}=c_{22} \pm \sqrt{\beta+c_{22}^{2}}
$$

and the resulting matrix functions $Z=Q_{*} Y Q$ and $M Q_{*}$ (cf. (3.9)) are

$$
Z=\left[\begin{array}{cc}
0 & 0 \\
0 & Y_{22}
\end{array}\right], \quad M Q_{*}=\left[\begin{array}{cc}
0 & 0 \\
0 & c_{22}-Y_{22}
\end{array}\right] .
$$


In this case the conditions (3.25) and (3.26) are equivalent to the conditions that

$$
\begin{gathered}
Y_{22}(t) \text { has no zeros, and } \\
Y_{22}(t)-c_{22}(t)= \pm \sqrt{\beta+c_{22}(t)^{2}} \text { has no zeros, respectively. }
\end{gathered}
$$

Case I: $c_{12}$ and $c_{21}$ vanish identically, $\beta>0$.

Here, both, $Y_{22}$ and $Y_{22}-c_{22}$ do not have zeros, i.e., the conditions (3.25) and (3.26) are fulfilled. Equation (5.5) is simply $0=\left(Y_{22}-c_{22}\right) Y_{21}$, which leads to $Y_{21}=0$. (5.6) yields $Y_{22}^{\prime}=-\alpha$. Hence, in this case

$$
Y(t)=\left[\begin{array}{cc}
-\alpha(t-1) & 0 \\
0 & c_{22}(t) \pm \sqrt{\beta+c_{22}(t)^{2}}
\end{array}\right]
$$

solves the Riccati-DAE system. The feedback optimal control is $u=-\left(c_{22} \pm \sqrt{\beta+c_{22}^{2}}\right) x_{2}$. The optimal trajectory, i.e., the solution of the $\operatorname{IVP}(4.1),(4.2)$ (i.e., $(2.13))$ is $x_{*}(t) \equiv$ $\left(\begin{array}{c}x_{10} \\ 0\end{array}\right)$, the optimal control is $u_{*}=0$, and the optimal cost $J\left(u_{*}, x_{*}\right)=\frac{1}{2} \alpha x_{10}^{2}$.

Case II: $c_{22}$ vanishes identically, $c_{12}$ and $c_{21}$ have no zeros, $\beta>0$. Again, both, $Y_{22}=$ $\pm \sqrt{\beta}$ and $Y_{22}-c_{22}=Y_{22}$ have no zeros, and the conditions (3.25) and (3.26) are fulfilled. This time, equation (5.5) leads to

$$
Y_{21}=c_{21} \pm \frac{1}{\sqrt{\beta}} c_{12} Y_{11}
$$

From (5.6) and (5.13) we derive the ODE

$$
Y_{11}^{\prime}=-\alpha-2 c_{21}\left(c_{21} \pm \frac{1}{\sqrt{\beta}} c_{12} Y_{11}\right)+\left(c_{21} \pm \frac{1}{\sqrt{\beta}} c_{12} Y_{11}\right)^{2}
$$

For example, for $c_{12}=c_{21}=1, x_{10}=1$ it results that

$$
\begin{aligned}
Y_{11}^{\prime} & =-(\alpha+1)+\frac{1}{\sqrt{\beta}} Y_{11}^{2}, \\
Y_{11}(t) & =\beta \gamma \frac{1-e^{2 \gamma(t-1)}}{1+e^{2 \gamma(t-1)}}, \quad \text { with } \gamma=\sqrt{\frac{1+\alpha}{\beta}} .
\end{aligned}
$$

Then, $u=\left(\mp \frac{1}{\sqrt{\beta}} Y_{11}-1\right) x_{1} \mp \sqrt{\beta} x_{2}$ is the optimal feedback control. The DAE (4.1) is of the form

$$
x_{1}^{\prime}=x_{2}, \quad 0=\frac{1}{\sqrt{\beta}} Y_{11} x_{1}+\sqrt{\beta} x_{2},
$$

the optimal pair $\left(u_{*}, x_{*}\right)$ consists of

$$
u_{*}(t)=-x_{* 1}(t), \quad x_{* 1}(t)=\frac{e^{\gamma t}+e^{\gamma(2-t)}}{1+e^{2 \gamma}} z_{0}, \quad x_{* 2}(t)=-\frac{1}{\beta} Y_{11}(t) x_{* 1}(t) .
$$

The minimal cost is $J\left(u_{*}, x_{*}\right)=\frac{\beta \gamma}{2} \cdot \frac{1-e^{-2 \gamma}}{1+e^{-2 \gamma}}$.

Case III: $\beta=0, c_{22}$ vanishes identically, $c_{12}, c_{21}$ have no zeros. Here, (5.9) implies $Y_{22}=0$, hence, $Z=0, M Q_{*}=0$, and the conditions (3.25) and (3.26) fail to be valid. 
Equation (5.5) simplifies to $c_{12} Y_{11}=0$, hence $Y_{11}=0$ must be true. By equation (5.6) we find $Y_{21}=c_{21} \pm \sqrt{\alpha+c_{21}^{2}}$. Therefore, the matrix function

$$
Y=\left[\begin{array}{cc}
0 & 0 \\
c_{21} \pm \sqrt{\alpha+c_{21}^{2}} & 0
\end{array}\right]
$$

solves the system (3.1)-(3.3), however, the conditions (3.25) and (3.26) do no longer hold. The resulting DAE (4.1) is now $x_{1}^{\prime}=c_{12} x_{2}, 0=\sqrt{\alpha+c_{21}^{2}} x_{1}$, and it has the trivial solution only. Consequently, for $x_{10} \neq 0$, there is no solution of the $\operatorname{IVP}(4.1),(4.2)$. If $x_{10}=0$, then the trivial pair $u_{*}=0, x_{*}=0$ is the optimal one in accordance with Theorem 2.5. If $x_{10} \neq 0$, then the linear quadratic optimal control problem has no solution at all.

The so-called Hamiltonian system (cf. Section 6 below) corresponding to the special problem (5.1)-(5.3) is the following one:

$$
\begin{aligned}
x_{1}^{\prime} & =c_{12} x_{2}, \\
0 & =c_{21} x_{1}+c_{22} x_{2}-\psi_{2}, \\
-\psi_{1}^{\prime} & =\alpha x_{1}+c_{21} \psi_{2}, \\
0 & =\beta x_{2}+c_{12} \psi_{1}+c_{22} \psi_{2} .
\end{aligned}
$$

For this system, the boundary conditions

$$
x_{1}(0)=x_{10}, \quad \psi_{1}(1)=0,
$$

have to be taken into accout. This linear DAE with respect to $x, \psi$ is regular with index one exactly if $\beta+c_{22}^{2} \neq 0$. This index-one conditon is valid in Case I and Case II.

In Case III, the boundary value problem (5.14),(5.15) has no solution for $x_{10} \neq 0$. For $x_{10}=0$ it has the trivial solution. It may be checked that this DAE has index two.

Notice that for the solvability of the corresponding Riccati DAE (1.10) it is necessary that $\beta=0$ is given (cf. [KuMe]), i.e., this Riccati DAE is no longer solvable in the unproblematic cases I and II. In the case III the final value problem for the Riccati DAE (1.10) may or may not have solutions. From this point of view, the Riccati DAEs (1.7) or (1.10) seem not to be appropriate tools for constructing optimal feedback solutions, whereas the Riccati DAE (1.15) and the versions in [Ku1], [Ku2], [Ku3], [KlKu] are useful for this purpose.

\section{Riccati equations and Hamiltonian systems}

Theorem 6.1 Given a solution $Y$ of (2.7),(2.8) with $A^{*} Y Q=0$. If the continuous matrix function $X:[0, T] \rightarrow L\left(\mathbb{R}^{p}, \mathbb{R}^{m}\right)$ having a $C^{1}$-part $B X$ satisfies the equation

$$
A(B X)^{\prime}=\left(C-D R^{-1} S^{*}-D R^{-1} D^{*} Y\right) X,
$$

then the pair $X, \Psi:=Y X$ forms a solution of the Hamiltonian system

$$
\begin{gathered}
A(B X)^{\prime}=\left(C-D R^{-1} S^{*}\right) X-D R^{-1} D^{*} \Psi \\
-B^{*}\left(A^{*} \Psi\right)^{\prime}=\left(W-S R^{-1} S^{*}\right) X+\left(C^{*}-S R^{-1} D^{*}\right) \Psi .
\end{gathered}
$$

$\Psi$ is continuous with $A^{*} \Psi$ being continuously differentiable. 


\section{Proof:}

(6.2) is a trivial consequence of (6.1).

Due to $A^{*} \Psi=A^{*} Y X=A^{*} Y B^{-} B X, A^{*} \Psi$ is continuously differentiable . We derive

$$
\begin{aligned}
B^{*}\left(A^{*} \Psi\right)^{\prime} & =B^{*}\left(A^{*} Y B^{-}\right)^{\prime} B X+B^{*} A^{*} Y B^{-}(B X)^{\prime} \\
& =B^{*}\left(A^{*} Y B^{-}\right)^{\prime} B X+Y^{*} A(B X)^{\prime}=-\left(W-S R^{-1} S^{*}\right) X-\left(C^{*}-S R^{-1} D^{*}\right) \Psi
\end{aligned}
$$

The above pair $X, \Psi$ combines $p$ columns of solutions of the DAE

$$
\left[\begin{array}{cc}
A & 0 \\
0 & -B^{*}
\end{array}\right] \frac{d}{d t}\left(\left[\begin{array}{cc}
B & 0 \\
0 & A^{*}
\end{array}\right]\left[\begin{array}{l}
x \\
\psi
\end{array}\right]\right)=\left[\begin{array}{cc}
C-D R^{-1} S^{*} & -D R^{-1} D^{*} \\
W-S R^{-1} S^{*} & C^{*}-S R^{-1} D^{*}
\end{array}\right]\left[\begin{array}{l}
x \\
\psi
\end{array}\right]
$$

If one tries to solve the Hamiltonian system (6.2),(6.3) one is confronted with the solvability problem concerning the DAE (6.4), hence, with its index.

Notice that (6.4) has a properly stated leading term since (2.2) has it. (6.4) is a square system having $m+k$ equations resp. $m+k$ unknown functions.

Theorem 6.2 If $A$ and $B$ remain nonsingular, equation (6.4) represents an implicit regular ODE (regular DAE with tractability index zero). Otherwise, for the DAE (6.4), it is necessary and sufficient to be regular with tractability index one, that the following two conditions are satisfied

$$
\begin{gathered}
{[A B-C Q, D] \quad \text { has full row rank } k} \\
i m\left[Q\left(C^{*}-S R^{-1} D^{*}\right) Q_{*}, Q\left(W-S R^{-1} S^{*}\right) Q\right]=i m Q .
\end{gathered}
$$

\section{Proof:}

In case of nonsingular $A$ and $B$, the assertion is obvious. Let $A, B$ be singular. In [BaKuMa], the pair of conditions

$$
\begin{gathered}
{[A B-C Q, D] \quad \text { has full row rank } k \text {, and }} \\
{\left[\begin{array}{ccc}
B^{*} A^{*}-C^{*} Q_{*} & W Q & S \\
-D^{*} Q_{*} & S^{*} Q & R
\end{array}\right] \quad \text { has full row rank } m+l}
\end{gathered}
$$

was shown to be necessary and sufficient for the DAE

$$
\left[\begin{array}{cc}
A & 0 \\
0 & -B^{*} \\
0 & 0
\end{array}\right] \frac{d}{d t}\left(\left[\begin{array}{ccc}
B & 0 & 0 \\
0 & A^{*} & 0
\end{array}\right]\left[\begin{array}{l}
x \\
\psi \\
u
\end{array}\right]\right)=\left[\begin{array}{ccc}
C & 0 & D \\
W & C^{*} & S \\
S^{*} & D^{*} & R
\end{array}\right]\left[\begin{array}{l}
x \\
\psi \\
u
\end{array}\right]
$$

to be a regular one with tractabaility index one. Clearly, (6.9) is regular with index one if (6.4) is so, and vice versa. Hence, the above two conditions are valid for (6.4), too. The condition (6.7) coincides with (6.5). Taking into account the invertibility of $R$, the second condition (6.8) is equivalent to (notice that in $[\mathrm{BaKuMa}]$ slightly more general problems with $R$ positive semidefinite are considered $)$ the injectivity of $\left[\begin{array}{c}A B \\ Q_{*}\left(C-D R^{-1} S^{*}\right) \\ Q\left(W-S R^{-1} S^{*}\right)\end{array}\right]$, but this is equivalent to (6.6). 
Remark 6.3 In [BeLa], descriptor systems (1.6) being in an SVD coordinate system play a special role, and in particular, the invertibility of a certain matrix $\bar{R}$ (cf. [BeLa]) is a basic property assumed to be given in all four versions of Riccati differential equations studied in [BeLa, $\S I V]$. From the viewpoint of DAE theory, for those very special systems (6.9), the invertibility of $\bar{R}$ exactly means regularity with tractability index one (cf. $[\mathrm{BaKuMa}])$.

Remark 6.4 Recall from [BaKuMa] that, if the system (6.9) is regular with tractability index one, and, additionally, $\operatorname{ker} B^{*}=0$, then the so-called inherent regular ODEs of (6.9) and of (6.2),(6.3), respectively, actually have a Hamiltonian structure - a property that should be useful concerning the solvability of boundary value problems for the Hamiltonian system (6.2),(6.3). However, notice that in general it may happen that the so-called Hamiltonian system (6.2),(6.3) may lose the inherent Hamiltonian structure (cf. $[\mathrm{BaKuMa}])$.

We end up with an assertion saying that if the Hamiltonian system (6.2),(6.3) has a somehow good solvability, then the Riccati-DAE system (3.1),(3.2) is solvable at the same time.

Theorem 6.5 Let $X \in C\left([0, T], L\left(\mathbb{R}^{m}\right)\right), \Psi \in C\left([0, T], L\left(\mathbb{R}^{m}, \mathbb{R}^{k}\right)\right)$ be such that their $m$ columns belong to $C_{B}^{1}\left([0, T], \mathbb{R}^{m}\right)$, resp. $C_{A^{*}}^{1}\left([0, T], \mathbb{R}^{k}\right)$ and

$$
\begin{gathered}
A(B X)^{\prime}=\left(C-D R^{-1} S^{*}\right) X-D R^{-1} D^{*} \Psi \\
-B^{*}\left(A^{*} \Psi\right)^{\prime}=\left(W-S R^{-1} S^{*}\right) X+\left(C^{*}-S R^{-1} D^{*}\right) \Psi
\end{gathered}
$$

is satisfied.

Let $X$ be nonsingular and let $X^{-1} B^{-}$belong to $C^{1}$. Let $Y:=\Psi X^{-1}$ be such that

$$
P_{*} Y Q=0, \quad A^{*} Y B^{-}=B^{-*} Y^{*} A .
$$

Then, $Y$ is continuous with a continuously differentiable part $A^{*} Y B^{-}$and satisfies the Riccati-DAE system (3.1),(3.2).

\section{Proof:}

Here condition (3.2) is given, and $A^{*} Y B^{-}=A^{*} Y X X^{-1} B^{-}=A^{*} \Psi X^{-1} B^{-}$belongs to $C^{1}$. Derive from (6.11)

$$
B^{*}\left(A^{*} Y X\right)^{\prime} X^{-1}=-\left(W-S R^{-1} S^{*}\right)-\left(C^{*}-S R^{-1} D^{*}\right) Y
$$

By means of

$B^{*}\left(A^{*} Y X\right)^{\prime} X^{-1}=B^{*}\left(A^{*} Y B^{-} B X\right)^{\prime} X^{-1}=B^{*}\left(A^{*} Y B^{-}\right)^{\prime} B+B^{*} A^{*} Y B^{-}(B X)^{\prime} X^{-1}=$ $B^{*}\left(A^{*} Y B^{-}\right)^{\prime} B+Y^{*} A(B X)^{\prime} X^{-1}$ and taking into account (6.10), (6.11) we obtain (3.1).

If $X, \Psi$ in Theorem 6.5 are chosen to fulfil the final conditions $B(T)^{*} A(T)^{*} \Psi(T)=$ $V, A(T) B(T) X(T)=A(T) B(T)$ then it follows that $A(T)^{*} Y(T) B(T)^{-}=$ $=B(T)^{-*} V B(T)^{-}$; i.e., (3.3) is satisfied. 


\section{Final Remark}

We have shown that optimal feedback controls of linear-quadratic optimal control problems with constraints described by general linear DAEs with variable coefficients can be computed by suitably formulating a Riccati DAE, similarly to the classical example where the constraints are described by explicit ODEs.

Compared to earlier papers we could do without several, partly very restrictive assumptions. Furthermore, it is not necessary and probably not even reasonable to transform the DAE determining the constraints (descriptor system) or the DAE describing the Hamiltonian system into a special form with great expense.

What is on the agenda is the development of feasible solution methods for the Riccati DAE (3.1),(3.2).

\section{References}

[A-K et all] H. Abou-Kandil, G. Freiling, V. Ionescu, G. Jank: Matrix Riccati Equations in Control and Systems Theory. Birkhäuser Verlag, Basel-Boston-Berlin, 2003.

[BaKuMa] K. Balla, G.A. Kurina, R. März: Index criteria for differential algebraic equations arising from linear-quadratic optimal control problems. Institut für Mathematik, Humboldt-Universität zu Berlin, Preprint 14, 2003, www.mathematik.huberlin.de/publ/pre/2003/p-list-03.html

[BaMa] K. Balla, R. März: A unified approach to linear differential algebraic equations and their adjoints. Zeitschrift f. Analysis und ihre Anwendungen 21(2002)3, pp. 783-802.

[BeLa] D.J. Bender, A.J. Laub: The linear-quadratic optimal regulator for descriptor systems. IEEE Transactions on Automatic Control (1987), vol. AC-32(8), pp. 672-688.

[CaMa] S.L. Campbell, R. März: Characterization and computation of a "good control". Institut für Mathematik, Humboldt-Universität zu Berlin, Preprint 10, 2004, www.mathematik.hu-berlin.de/publ/pre/2004/p-list-04.html

[Do] H. Döring: Traktabilitätsindex und Eigenschaften von matrixwertigen RiccatiTyp Algebrodifferentialgleichungen. Diplomarbeit, Institut für Mathematik, Humboldt-Universität zu Berlin, 2004.

[KlKu] I.V. Klinskih, G.A. Kurina: Feedback control for a class of descriptor systems. Theory of evolution equations. International Conference Fifth Bogolyubov's reading. Abstracts of reports Karnyanets-Podilskyy, 2002, p.85 (in Russian).

[KuMa] G. A. Kurina, R. März: On linear-quadratic optimal control problems for timevarying descriptor systems. SIAM J. Control and Optimization, 42(6) (2004), pp. 2062-2077.

[KuMe] P. Kunkel, V. Mehrmann: The linear quadratic optimal control problem for linear descriptor systems with variable coefficients. Math.Control Signals Systems (1997) 10, pp. 247-264. 
[Ku1] G.A. Kurina: Design of feedback control for linear control systems unresolved with respect to derivative. Unpublished paper N 3619-82, VINITI, Voronezh, 1982 (in Russian).

[Ku2] G.A. Kurina: Feed-back control for linear systems unresolved with respect to derivative. Avtomatika i telemehanika (1984), no. 6, pp.37-41 (in Russian).

[Ku3] G.A. Kurina: On operator Riccati equation unresolved with respect to derivative. Differencial'nye uravnenija, (1986) vol. XXII, no. 10 , pp. 1826-1829 (in Russian).

[Ku4] G.A. Kurina: Singular perturbations of control problems with equation of state not solved for the derivative (A survey). Journal of Computer and System Sciences International (1993)31, pp. 17-45.

[Ku5] G.A. Kurina: Feed-back control for time-varying descriptor systems. Systems Science (2000), vol 26, no. 3, pp. 47-59 